\title{
Optical anisotropy induced by external magnetic fields on magnetic fluid films
}

\author{
S.Y. Yang ${ }^{\mathrm{a}, *}$, W.S. Tse ${ }^{\mathrm{a}}$, H.E. Horng ${ }^{\mathrm{b}}$, H.C. Yang ${ }^{\mathrm{c}}$, Chin-Yih Hong ${ }^{\mathrm{d}}$ \\ ${ }^{a}$ Institute of Physics, Academia Sinica, Taipei 115, Taiwan \\ ${ }^{\mathrm{b}}$ Department of Physics, National Taiwan Normal University, Taipei 116, Taiwan \\ ${ }^{\mathrm{c}}$ Department of Physics, National Taiwan University, Taipei 106, Taiwan \\ ${ }^{\mathrm{d}}$ Department of Mechanical Engineering, Da-Yeh University, Changhwa 105, Taiwan
}

\begin{abstract}
We examined the optical anisotropy of a magnetic fluid film under magnetic fields parallel to the plane of the film by detecting its birefringence effect. It was found that the transmission axis of the transmitted light was rotated significantly by an angle $\Delta \eta$, with respect to the axis of the linearly polarized incident light when an external magnetic field is applied. The angle $\Delta \eta$ increases with an increase of the field strength and then becomes saturated for higher fields. By comparing the detected $\Delta \eta$ 's with the corresponding structures formed in the magnetic fluid film under various magnetic fields, the angle $\Delta \eta$ was found to be proportional to the ratio of the chain area to the liquid area in the film. (C) 2001 Elsevier Science B.V. All rights reserved.
\end{abstract}

Keywords: Anisotropy — field induced; Magnetic fluids; Birefringence

\section{Introduction}

The study of magnetically induced birefringence of magnetic fluid films under magnetic fields parallel to the plane of the films (parallel magnetic fields) has attracted sustained interests [1-6]. There have been proposed some possible origins for this phenomenon, such as the nonsphericity of ferrite particles [1], needle-like chains [2] and anisotropic interaction between the aligned ferrite particles [3]. Recently, we found the long-chain structure in the magnetic fluid films under parallel magnetic fields [4] and observed directly significant Cotton-Mounton rotations. The two-phase theoretical model [5], in which the effects of incident light interacting with the long chains and the liquid phase are taken into account for the transmitted light, was then

\footnotetext{
* Correspondence address. Department of Physics, National Taiwan Normal University, 88, Sec. 4, Ting-Chou Rd., Taipei 116, Taiwan. Fax: + 886-2-293-09-439.

E-mail address: emesdolb@ms28.hinet.net (S.Y. Yang).
}

developed to interpret the results of the observed birefringence of the magnetic fluid films. A confirmation of the theoretical prediction and by the experimental data was obtained. This suggests that the birefringence of the magnetic fluid film is strongly correlated to the long chains. To directly clarify the role of the long chains in the birefringence effect, the numbers of the long chains in the magnetic fluid film were altered by changing the magnetic field strength and the corresponding CottonMounton rotation was measured.

\section{Experimental details}

The ferrofluid used is kerosene-based $\left(M_{\mathrm{s}}=\right.$ $6.4 \mathrm{emu} / \mathrm{g}$ ). It was sealed in a $4 \times 1 \mathrm{~cm}^{2}$ rectangular glass cell to form a $5 \mu \mathrm{m}$-thick film. The magnetic field $H$ was provided by a pair of solenoids and applied along the plane of the film. The details of observing the structures in the magnetic fluid film under external magnetic fields are reported elsewhere [6]. The experimental setup used to detect the birefringence is shown in Fig. 1. To obtain linearly polarized incident light, a laser beam $(\lambda=632.8 \mathrm{~nm})$ 


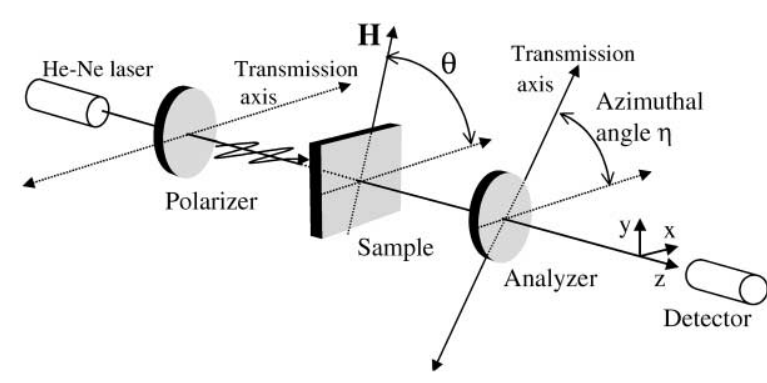

Fig. 1. Setup of the experiment for investigating the CottonMouton effect of the magnetic fluid film.

was passed through a linear polarizer which was normally incident to the film. After the incident light traveled through the film, the azimuthal distribution of the transmitted intensity was probed by using an analyzer to analyze the polarization of the transmitted light. The azimuthal angle, $\eta$, was scanned by using a step motor to rotate the transmission axis of the analyzer. It has been indicated that the most significant variation in the polarization of the incident light through the magnetic fluid occurs when the angle, $\theta$, between the $H$ and the polarization of the incident light is $45^{\circ}$ [5]. Hence, the angle $\theta$ is set at $45^{\circ}$ here. The other experimental details of detecting the birefringence are illustrated in Ref. [4].

\section{Results and discussion}

When applying a parallel magnetic field $H$ to the magnetic fluid film, some discrete magnetic short chains were formed because of the agglomeration of the magnetic particles. By increasing $H$ up to $30 \mathrm{Oe}$, we caused the short chains to join together and form longer chains. When $H$ was further raised, more chains were generated. A typical image of the long-chain structure is shown in Fig. 2 for 150 Oe. To reveal the magnetic-field-dependent long-chain structure, the ratio $r$ of the chain area to the liquid area was evaluated for each $H$ (shown in Fig. 2). The area occupied by the long chains is increased for higher $H$ 's and then becomes saturated as $H>120$ Oe.

With these long chains, a spatial anisotropy is induced in the magnetic fluid film under parallel $H$ 's. This spatial anisotropy causes a birefringence as a light linearly polarized is incident to the magnetic fluid film. To investigate the birefringence effect, $I-\eta$ curves of the transmitted light were measured under various $H$ 's at $\theta=45^{\circ}$. Under zero magnetic field, the $I-\eta$ curve was symmetric with a relative maximum intensity at $\eta_{I=\max }=0^{\circ}$ and zero intensity at $\eta_{I=\min }=90^{\circ}$ and $-90^{\circ}$. The symmetric $I-\eta$ curves under $H \neq 0$ were also obtained with a translational shift by a $\Delta \eta\left(\equiv\left|\eta_{I=\min }(H)-\eta_{I=\min }(H=0)\right|\right)$ with respect to the zero field curve. This shift implies that the

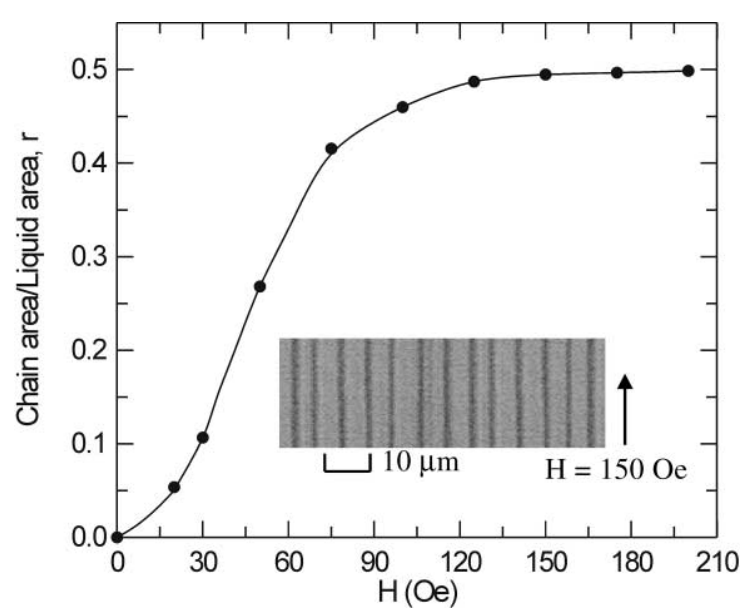

Fig. 2. Magnetic field dependence of the area ratio $r$ of the chain area to the liquid area. The inset shows the long-chain structure under $150 \mathrm{Oe}$.

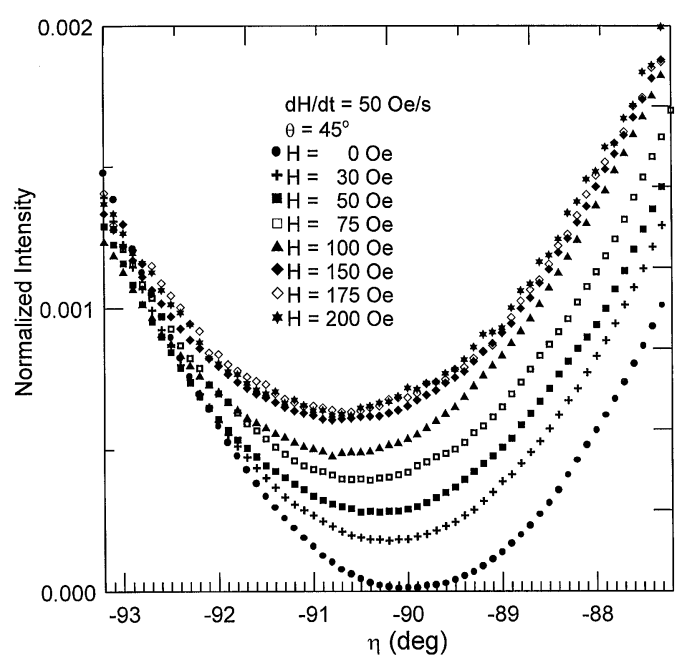

Fig. 3. $I-\eta$ curves under various magnetic fields at $\theta=45^{\circ}$.

transmission axis of the transmitted light was rotated (i.e. $\mathrm{C}-\mathrm{M}$ rotation) by an angle $\Delta \eta$ with respect to that of the incident light. To decide the $\Delta \eta$ precisely, the $I-\eta$ results at $\eta$ around $-90^{\circ}$ were enlarged and are shown in Fig. 3 . The angle $\eta_{I=\min }$ is moved with the increase of $H$ and then remains almost constant as $H>120 \mathrm{Oe}$. This gives a magnetic-field-dependent $\Delta \eta$ as shown in Fig. 4. The minimum intensity $I_{\min }$ occurred at $\eta_{I=\min }$ is nonzero under $H \neq 0$. That $I_{\min } \neq 0$ suggests that the transmitted light is not linearly but elliptically polarized.

Since the birefringence of the magnetic fluid film is attributed to the spatial anisotropy induced by the longchain structure, it is important to know the correlation between them. To do this, the $\Delta \eta-H$ curve in Fig. 4 is 


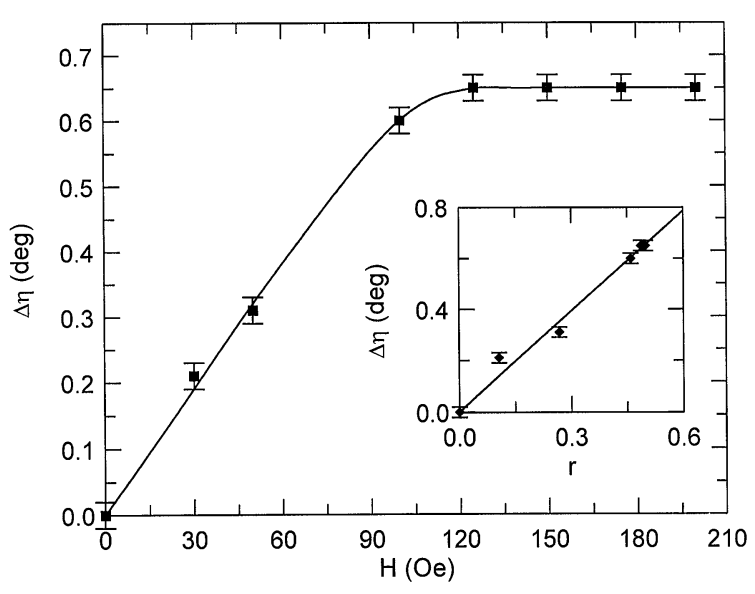

Fig. 4. C-M rotation angle $\Delta \eta$ versus the applied magnetic field. The inset is the relationship between the $\Delta \eta$ and $r$.

compared with the $r-H$ curve in Fig. 2. These two curves exhibit a similar trend. Furthermore, a linear relationship is obtained between $\Delta \eta$ and the area ratio $r$, shown as the inset in Fig. 4. This gives direct evidence that the birefrin- gence of the magnetic fluid film is due to the long chains. Also, the amounts of long chains dominate the $\mathrm{C}-\mathrm{M}$ rotation angle $\Delta \eta$.

\section{Conclusion}

The long chains formed in the magnetic fluid film under parallel magnetic fields lead to the birefringence effect. By altering the numbers of the long chains in the film resulted by changing the applied magnetic field, the $\mathrm{C}-\mathrm{M}$ rotation can be adjusted.

\section{References}

[1] A. Peterlin, H.A. Stuart, Z. Phys. 112 (1939) 129.

[2] S. Taketomi, Jpn. J. Appl. Phys. 22 (1983) 1137.

[3] M. Xu, P.J. Ridler, J. Appl. Phys. 82 (1997) 326.

[4] H.E. Horng et al., J. Magn. Magn. Mater. 201 (1999) 215.

[5] H.E. Horng et al., Magnitnaya Gidrodinaika 36 (1) (2000) 39.

[6] Chin-Yih Hong et al., J. Appl. Phys. 81 (1997) 4275. 